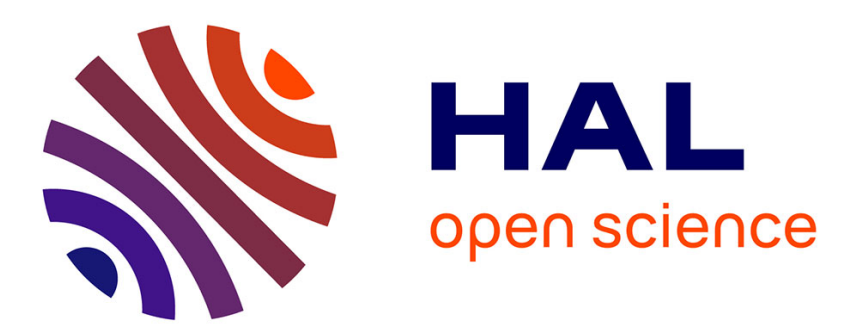

\title{
The Contribution concept for the control of a manufacturing multi-criteria performance improvement
}

Lamia Berrah, Vincent Clivillé, Gilles Mauris, Jacky Montmain

\section{To cite this version:}

Lamia Berrah, Vincent Clivillé, Gilles Mauris, Jacky Montmain. The Contribution concept for the control of a manufacturing multi-criteria performance improvement. Journal of Intelligent Manufacturing, 2019, 30 (1), pp.47-58. 10.1007/s10845-016-1227-9 . hal-01343905

\section{HAL Id: hal-01343905 \\ https://hal.science/hal-01343905}

Submitted on 3 Mar 2020

HAL is a multi-disciplinary open access archive for the deposit and dissemination of scientific research documents, whether they are published or not. The documents may come from teaching and research institutions in France or abroad, or from public or private research centers.
L'archive ouverte pluridisciplinaire HAL, est destinée au dépôt et à la diffusion de documents scientifiques de niveau recherche, publiés ou non, émanant des établissements d'enseignement et de recherche français ou étrangers, des laboratoires publics ou privés. 


\title{
The Contribution concept for the control of a manufacturing multi-criteria performance improvement
}

\author{
L. Berrah ${ }^{1}$ - V. Clivillé ${ }^{1}$ - J. Montmain ${ }^{2}$ - G. Mauris ${ }^{1}$
}

\begin{abstract}
By dealing with an overall manufacturing performance improvement context, we introduce in this paper the "improvement contribution" concept. A framework that integrates such a concept to the quantification of a multi-criteria interacting performance is proposed. The improvement contribution is defined as a new intelligent functionality that quantifies the impact of the improvement of a single (or a set of) mono-criterion performance(s) on the improvement of an overall performance. When performances are interacting, the quantification of such a contribution cannot be direct. The proposed approach consists of an extension of a previously developed Performance Measurement System (PMS). The considered PMS integrates an aggregation operator-the Choquet Integral (CI) - for the expression of an overall performance by handling weights and interactions between the mono-criterion performances. The principles of the improvement contribution and its quantification are thus presented in addition to the way the improvement contribution can be used for helping decision-makers in their manufacturing improvement control. As an illustration, the use of these contributions within successive iterations of improvement actions is shown using a case study submitted by the SME Fournier Company.
\end{abstract}

Fournier Company is a French manufacturer of kitchen and bathroom furniture.

V. Clivillé

vincent.cliville@univ-savoie.fr

1 LISTIC, Université Savoie Mont Blanc, BP 80439, 74944 Annecy le Vieux cedex, France

2 ENSTIMA Site EERIE - parc scientifique Georges Besse, 30035 Nîmes, France
Keywords Manufacturing performance - Improvement contribution - Multi-criteria and interacting performance . Performance measurement system - Choquet integral

\section{Introduction - Problem statement}

According to their context, manufacturing companies fix their strategies and define their objectives. The objectives are generally various and numerous, declared with regards to the success performance criteria of the companies. Once the objectives are declared, actions are planned and executed in order to achieve the objectives (Kaplan and Norton 1992). The results are then checked and decisions are taken, concerning the definition of new objectives, the reconfiguration of the business units or the manufacturing processes... These iterative steps are referred to as the well-known continuous performance improvement cycle, Plan-Do-Check-Act (PDCA) (Imai 1986; Ohno 1988).

Our study subscribes to the continuous improvement of an overall manufacturing performance. Such a performance can be seen either as an overall expression or as a vector of elementary performances. The overall performance becomes multi-criteria while the elementary performances are mono-criterion. Within this framework, the overall performance improvement can be achieved in numerous ways, namely according to different possible action plans. Indeed, an overall performance improvement that depends on a set of mono-criterion performance improvements can be achieved by enhancing the improvement of one single particular performance or a particular subset of such performances... To be more precise, the nature of the action, the performance on which it impacts, its ranking among all the planned actions, the number of times it is launched... all characterise an action plan and makes such a plan not unique. Action plans can 
also vary with regards to the decision-maker in charge of the improvement "philosophy". Hence, action plans are not necessarily similar, inducing for each of them, a particular strategy of improvement. One can prefer a regular balanced improvement while another looks for a quick and spectacular improvement with regards to a pre-selected mono-criterion performance. The choice of the improvement action plan thus depends on the preferences of the decision-maker as well as on the company context (Oztemel 2010).

According to all these considerations, the contribution concept, which is generally defined as: "the part played by a person or thing in bringing about a result or helping something to advance" (Oxford dictionary 2016) can be applied for the handling of the impact of the improvement of a set of mono-criterion performances into the overall performance improvement.

The aim of this study is specifically the identification, i.e. the definition and the quantification of such an improvement contribution. Even if it is intuitive, the use of the contribution notion for manufacturing control or, more widely, for decision-making is rather new. By assuming that PMS's (Performance Measurement Systems) are the tools, that are dedicated to provide the useful performance pieces of information for the control, we present hereafter a brief analysis of what is proposed to support control for a continuous performance improvement. Note that other approaches for the manufacturing control that are also related to multi-criteria decision-making support can be found in the literature ${ }^{1}$.

The paper's propositions put forward the concept of improvement contribution to complement a previously developed PMS able to take performance interactions into account. Formally, the approach is based on the handling of the Choquet Integral (CI) aggregated performance model. Hence, the paper is organised as follows. We begin first by a summarised state of the art concerning the PMS functionalities in terms of manufacturing improvement control. The results of this analysis as well as the assumptions that are necessary for our framework are then discussed. Then, after having intuitively illustrated the improvement contribution concept, some notations as well as a generic definition of the improvement contribution notion are proposed in "The improvement contribution quantification" section. We will focus on the quantification of the improvement contribution, even if the background on the specificities of the CI performance aggregation model is presented in Appendix 1. The aim of "Intelligent control using the improvement contribution" section is to highlight the use of the improvement contribution. The improvement contribution hence becomes

\footnotetext{
1 Recently the Journal of Intelligent Manufacturing have published several articles in this area (see e.g. (Bosch-Mauchand et al. 2012; Ounnar and Pujo 2012; Kocaoğlu et al. 2013; Shah et al. 2016; Singh et al. 2015; Gallasso et al. 2016))
}

an interactive element with a decision-maker for his improvement control. A simplified procedure is proposed in this sense. "Case study" section is dedicated to an improvement problem submitted by the Fournier Company. In this industrial case, the improvement contributions are quantified, respectively a priori and a posteriori of the improvement enactment. The a priori quantification is used in to plan the mono-criterion performances to achieve while the a posteriori quantification is used to make a diagnosis and reward the teams according to the achieved results. Finally some perspectives for further works are suggested.

\section{State of the art and research assumptions}

\section{The performance measurement systems}

The PMS's were introduced, in the mid-eighties, in order to integrate the numerous performance indicators that were progressively defined in manufacturing companies, into an overall multi-criteria framework (Globerson 1985; Neely et al. 1995; Kaplan and Norton 1996). The aim of the PMS's was to handle, define and quantify performances that were only financial and computed through Taylorian ratios (Johnson 1975). PMS's have been commonly defined as being a set of performance measures that are organised in order to handle the strategy of the company throughout the executive processes (Ghalayini et al. 1997; Kocaoğlu et al. 2013). The main PMS functionalities concern the quantification of both the efficiency and effectiveness of the improvement actions (Neely et al. 1995) and the performance processes monitoring, in addition to the accounting, the performance diagnosis, the team motivation enhancement and communications improvement (Waggoner et al. 1999). Among the numerous PMS's frameworks and models that have been proposed in the literature (Nudurupati et al. 2011; Shah et al. 2016), we consider hereafter the propositions that are more or less concerned with the improvement of overall performances. Knowing that all these propositions handle the overall, multi-criteria and interacting aspects of the performance, a summarised description of each PMS will allow us to have an idea of the pieces of information or functionalities that are provided for the overall performance improvement.

Neely and his team expose the general PMS principles in the Process Based Approach (PBA) (Bourne et al. 2000; Waggoner et al. 1999). In this framework, interactions are viewed as links between result indicators (that inform the management about the objectives achievement) and process indicators (that aid the management to control the launched actions). The supplied improvement performance information consists of numerical quantified objectives and measures in both the result and process indicators. The main PBA functionality in the performance improvement field can be seen 
through a type of network that links, throughout the improvement process, in a cognitive way, the achieved results with the in-progress actions.

The IDPMS (Integrated Dynamic Performance Measurement System) (Ghalayini 1996; Ghalayini et al. 1997) proposes a framework for controlling the performance improvement in a progressive way (Schneidermann 1988). Two types of interactions are identified, between the socalled Key Success Factors indicators and the Key Process Factors indicators. The subordination link is a vertical interaction between the indicators of different management levels (strategic, improvement and operational). The influence link is a horizontal interaction (positive or negative) between the indicators of a given management level. A performance aggregation model is proposed for quantifying the subordination link. The influence links are seen as "gains" or "losses" of given indicators with regards to others, for a given level (e.g. a given indicator increase of $x \%$ is a consequence of an increase of $y \%$ on another indicator). For a given improvement, the IDPMS supplies, on the one hand, both numerical quantified objectives and measures, for each indicator of the PMS, and, on the other hand, the set of numerical gains or losses according to the set of indicators. The main IDPMS functionality is thus a cause-effect model linking the improvement actions and the set of interacting indicators. Moreover, we want to mention that Ghalayini's team has highlighted, for the first time, the notion of impact of one performance versus another.

The famous BSC (Balanced Scorecard) (Kaplan and Norton 1992) has been introduced as a strategic management tool that describes a company according to four performance axes (Financial, Customer, Internal Processes, Learning and Growth). The company strategy is deployed under a set of BSC's, up to the operational decision level, and according to the different business units. At the operational level, an "initiative", namely an action, is systemically associated with each objective and thus each indicator. The interactions, between the initiatives, are defined and cognitively handled in the "strategic map" (Kaplan and Norton 1996). Moreover, a temporal break-down of the long-term objectives is proposed in the BSC, associating in this sense a set of "targets" for each objective. These targets are defined at specific milestones. Objectives, measures and targets are thus numerically quantified. The main improvement functionalities of the BSC are respectively the deployment of the strategy up to the initiative definitions, the temporal break-down of the objectives and the axes interaction identification.

In another way, the QMPMS (Quantified Models for Performance Measurement System) (Bititci 2001; Suwignjo and Bititci 2000) proposes to quantify the company multi-criteria performance in a normalised way. The cognitive map tool is used for representing respectively "self-interactions", "hierarchical" interactions and "mutual" interactions, between indicators. The weight of each indicator, with regards to the overall performance, is thus deduced, independently first and then conjointly with the other indicators. Elementary and aggregated normalised performances (called "quantified" performances by the authors) are provided thanks to the AHP (Analytic Hierarchy Process) method (Saaty 2000). This normalisation is based on a questionnaire for the gathering of expert knowledge. The main QMPMS functionality is a formal unified framework for expressing both monocriterion interacting performances and overall performances in an aggregative way. However, the use of the proposed framework in an improvement context is not highlighted.

Incidentally, by subscribing to the QMDPMS and the BSC philosophies, our previous works have focused on an aggregation performance model. In (Clivillé et al. 2007), a systemic view of a PMS was proposed, leading to a break-down of overall multi-criteria objectives into elementary mono-criterion objectives. A performance quantification mechanism was proposed. In a similar logic as AHP, monocriterion and multi-criteria performances are normalised thanks to the MACBETH (Multi Attractiveness Categorical based Evaluation TecHnique) method (Bana e Costa et al. 2012). Mutual interactions between criteria are taken into account thanks to the CI operator (Grabisch 1996). The performance quantification is done in an interactive way with the expert. The main functionality of this PMS is a formal unified framework for quantifying both monocriterion interacting performances and overall performance. Such a framework has been used to optimising performance improvement by quantifying efficacy and efficiency of the improvement (Berrah et al. 2011).

\section{Research assumptions}

Let us recall that we look, in this study, for information about the contribution of the improvement of one or a set of mono-criterion performance(s) on the improvement of the overall performance. From this view, according to the previous PMS's state of the art, we highlight the following key points:

- the overall, multi-criteria and interaction aspects of the performance are well-admitted;

- the provided performance pieces of information are numerically quantified;

- the weight of a mono-criterion performance in the overall performance is dealt with either in a cognitive way (PBA, BSC, IDPMS) or through a performance aggregation model (QMPMS, our proposition).

The aim of this work is to quantify the improvement contribution notion as introduced before. We make two major 
assumption families in this sense. The first assumption family concerns the performance quantification and the second assumption family concerns the PDCA improvement cycle.

From the performance quantification point of view, four assumptions are at the origin of the used framework.

(A1) The manufacturing performances are numerically quantified on $[0,1]$.

(A2) The multi-criteria performance is overall and thus broken-down into a vector of mono-criterion performances.

(A3) The multi-criteria performance is quantified by aggregating the mono-criterion performances.

(A4) The mono-criterion performances are interacting. From the performance improvement point of view, five assumptions are made.

(A5) The improvement cycle evolves throughout a series of states. In particular, state A identifies the beginning of an improvement cycle (i.e.a new iteration of it). State $\mathrm{B}$ identifies the end of the cycle (i.e. the considered iteration). Intermediate states can exist between $\mathrm{A}$ and B (during the $D o$-step).

(A6) A state is described by the vector of corresponding mono-criterion performances as well as the overall performance.

(A7) The performance improvement is introduced as being the gap between two successive performances that are associated with the corresponding states.

(A8) The improvement performance is always positive, possibly null.

(A9) By subscribing to the PDCA cycle, the performance pieces of information are provided in the Check step and used in the Act step.

\section{The improvement contribution quantification}

In this section, we begin by giving an illustration of the improvement contribution. Thus, we state some definitions and notations and then we present our proposition.

\section{Illustration of the improvement contribution notion}

By considering the previous assumptions, let us see an intuitive illustration of the improvement contribution notion. In the following example, mono-criterion performances of state $\mathrm{A}$ and their respective associated improvements that are achieved in state B are seen in Fig. 1.

Let us imagine that we want to quantify the contribution of a given mono-criterion performance improvement to the overall performance improvement, at the end of the considered iteration. By using the Weighted Arithmetic Mean

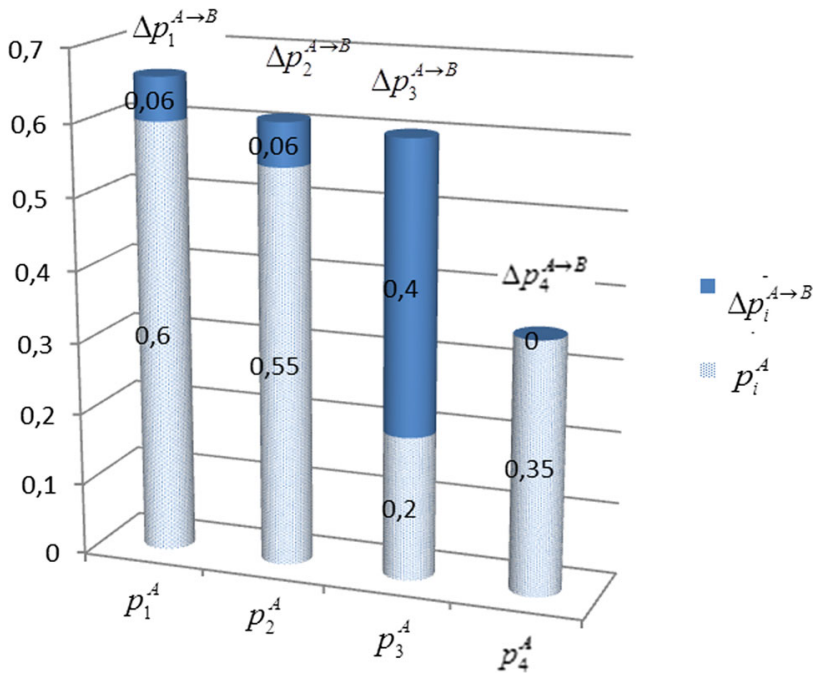

Fig. 1 Mono-criterion performances and associated improvement values for a WAM

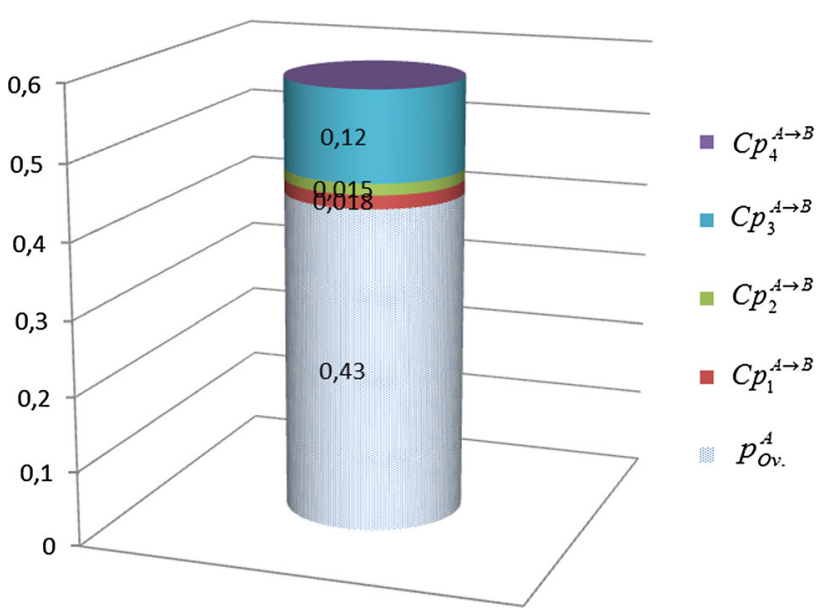

Fig. 2 Mono-criterion performance improvement contributions for a WAM

(WAM), the overall performance is the result of the weighted sum of the mono-criterion performances. Hence, the contribution of the improvement of a mono-criterion performance is no more than the product of such an improvement by the weight of the associated mono-criterion performance (see Fig. 2).

We choose to develop the improvement contribution concept in the same logic of this example, for a CI performance aggregation model that handles the interactions between the performances (beyond the weights). The aim is to make the quantification as simple as for the WAM.

\section{A definition for the improvement contribution}

Let $N$ be the set of the mono-criterion performances that are involved in the considered improvement cycle $(|N|=n)$. 
Let $E_{i}$ be the universe of discourse ([0,1] in our case) of the mono-criterion performance $p_{i}^{\alpha} \quad i=1$ to $n$ at state $\alpha$ (of the series of the improvement cycle states (cf. assumption (A5) §2.2). Let $\vec{P}^{\alpha}=\left(p_{1}^{\alpha} \ldots p_{n}^{\alpha}\right)$ be the corresponding monocriterion performances vector (cf. assumption (A2) § 2.2).

We respectively denote $\vec{P}^{A}=\left(p_{1}^{A} \ldots p_{n}^{A}\right)$ and $\vec{P}^{B}=$ $\left(p_{1}^{B} \ldots p_{n}^{B}\right)$ the mono-criterion performance vectors that correspond to states $\mathrm{A}$ and $\mathrm{B}$, of the considered improvement. The gap between these performances (cf. assumption (A7) $\S 2.2$ ) is respectively denoted $\Delta p_{i}^{A \rightarrow B}=p_{i}^{B}-p_{i}^{A}$ and $\Delta p_{O v .}^{A \rightarrow B}=p_{O v .}^{B}-p_{O v}^{A}$, with $p_{i}^{B} \geq p_{i}^{A}$ and $p_{O v}^{B} \geq p_{O v}^{A}$. (cf. assumption (A8) § 2.2).

Let $C_{K}^{A \rightarrow B}$ be the improvement contribution of a subset $K$ ( $K \subset N$ and $|K|=k$ ) of mono-criterion performances to the overall performance improvement. From a general point of view, we consider the quantification of such an improvement contribution as being a relation depending upon $K, \vec{P}^{A}$ and $\vec{P}^{B}$. When the aggregation operator is the WAM:

$C_{K}^{A \rightarrow B}=\sum_{i=1}^{k} w_{i}\left(p_{i}^{B}-p_{i}^{A}\right)$

where $w_{i}$ are the weights of the mono-criterion performances to the overall performance $\left(\sum w_{i}=1\right)$.

But as we have mentioned before, the quantification of $C_{K}^{A \rightarrow B}$ is based on a performance aggregation model that handles the interactions between the mono-criterion performances. According to previous works and to the assumptions (A3, A4) given in $\S 2.2$, the CI operator is considered here. For the sake of reading ease, the basics of this aggregation operator are recalled in Appendix 1. Two forms of CI are used, the initial form that highlights the interactions between the mono-criterion performances, and the piecewise linear form that is similar to the WAM writing.

\section{Improvement contribution quantification}

The quantification of the improvement contribution will concern the improvement from state A to state B of the considered improvement. However, the use of the CI performance aggregation model, in its piecewise linear form, can require the introduction of intermediate states between $\mathrm{A}$ and B (see Appendix 1 and assumption (A5) cf. § 2.2).

In order to introduce our model, we choose to distinguish three cases, two particular cases and the general case:

- All the mono-criterion performance improvement contributions are considered, $|K|=n$.

- Only one mono-criterion performance improvement contribution is considered, $|K|=1$.

- Several mono-criterion performance improvement contributions are considered, $1 \leq|K| \leq n$.
Note that the propositions are illustrated by examples that are extracted from the case study of this work $(\S 5)$.

Quantification of $C_{K}^{A \rightarrow B}$ for $K=N$

In this case, $C_{N}^{A \rightarrow B}=\Delta p_{O v}^{A \rightarrow B}=p_{O v}^{B},-p_{O v}^{A}$.

Example 1 Let us consider the CI aggregation model whose coefficients are given in Table 1 . Let us consider the case where $\vec{P}^{A}=(0.6,0.55,0.2,0.35)$ and $\vec{P}^{B}=(0.6,0.7,0.5$, $0.5)$. The associated overall performances are obtained by using (4) (See Appendix 1): $p_{O v}^{A}=0.364, p_{O v}^{B}=0.555$.

$$
\text { So } C_{N}^{A \rightarrow B}=p_{O v .}^{B}-p_{O v}^{A}=0.555-0.364=0.191 \text {. }
$$

Quantification of $C_{K}^{A \rightarrow B}$ for $|K|=1$

We consider here the quantification of only one improvement contribution when many mono-criterion performances are improved. It raises some questions because $\mathrm{CI}$ is not fully but only piecewise linear. Hence, the improvement contribution cannot be obtained, in the same way as using a WAM (1). However, by considering intermediate states between A and B, which correspond to linear form of CI, $C_{K}^{A \rightarrow B}$ will be obtained by making the sum of the improvement contributions that are achieved throughout all the considered states, between A and B. For the sake of simplicity, let us denote $\alpha, \ldots, \beta$ the intermediate states between $\mathrm{A}$ and $\mathrm{B}$, and $w_{i}^{A \rightarrow \alpha} \ldots w_{i}^{\beta \rightarrow B}$ the corresponding weights of the linear form of CI, then we have :

$$
\begin{aligned}
C_{K}^{A \rightarrow B} & =C_{K}^{A \rightarrow \alpha}+\cdots+C_{K}^{\beta \rightarrow B} \\
& =w_{i}^{A \rightarrow \alpha}\left(p_{i}^{\alpha}-p_{i}^{A}\right)+\cdots+w_{i}^{\beta \rightarrow B}\left(p_{i}^{B}-p_{i}^{\beta}\right)
\end{aligned}
$$

Note that such a vision amounts to knowing the intermediate states between $\mathrm{A}$ and $\mathrm{B}$.

Example 2 Let us consider again the preceding example where the mono-criterion performances are improved, from state $\mathrm{A}, \vec{P}^{A}=(0.6,0.55,0.2,0.35)$ to state $\mathrm{B}, \vec{P}^{B}=$ $(0.6,0.7,0.5,0.5)$. In addition, we consider the intermediate states $\alpha$ and $\beta: \vec{P}^{\alpha}=(0.6,0.55,0.35,0.35), \vec{P}^{\beta}=$ $(0.6,0.6,0.5,0.35)$.

Between state A and state $\alpha$, the mono-criterion performances weights become ${ }^{2}: w_{1}=0.05, w_{2}=0.4, w_{3}=$ $0.525, w_{4}=0.025$.

Between state $\alpha$ and state $\beta$, the mono-criterion performances weights become: $w_{1}=0.05, w_{2}=0.4, w_{3}=$ $0.275, w_{4}=0.275$.

\footnotetext{
2 The CI parameters are given in Table 1, the computation of the weights is given in Appendix 1.
} 
Table 1 CI coefficients for the Fournier Company case

\begin{tabular}{ccccccccc}
\hline CI coefficients & $\phi_{1}$ & $\phi_{2}$ & $\phi_{3}$ & $\phi_{4}$ & $I_{12}$ & $I_{13}$ & $I_{34}$ & Others $I_{i j}$ \\
\hline & 0.30 & 0.25 & 0.30 & 0.15 & 0.30 & 0.20 & 0.25 & 0 \\
\hline
\end{tabular}

Between state $\beta$ and state $\mathrm{B}$, the mono-criterion performances weights become: $w_{1}=0.35, w_{2}=0.1, w_{3}=$ $0.275, w_{4}=0.275$. Thus,

$$
\begin{aligned}
C_{1}^{A \rightarrow B} & =C_{1}^{A \rightarrow \alpha}+C_{1}^{\alpha \rightarrow \beta} \\
+C_{1}^{\beta \rightarrow B} & =0.05 \times 0+0.05 \times 0+0.35 \times 0=0, \\
C_{2}^{A \rightarrow B} & =C_{2}^{A \rightarrow \alpha}+C_{2}^{\alpha \rightarrow \beta} \\
+C_{2}^{\beta \rightarrow B} & =0.05 \times 0+0.4 \times 0.05+0.1 \times 0.1=0.03,
\end{aligned}
$$

$C_{3}^{A \rightarrow B}=C_{3}^{A \rightarrow \alpha}+C_{3}^{\alpha \rightarrow \beta}+C_{3}^{\beta \rightarrow B}=0.525 \times 0.15+$ $0.275 \times 0.15+0.275 \times 0=0.12$ and $C_{4}^{A \rightarrow B}=C_{4}^{A \rightarrow \alpha}+$ $C_{4}^{\alpha \rightarrow \beta}+C_{4}^{\beta \rightarrow B}=0.275 \times 0+0.275 \times 0.15=0.041$.

Note that $C_{1}^{A \rightarrow B}=0$, since $p_{1}$ has not been improved $p_{1}^{B}=p_{1}^{A}$.

Thus, we can conclude that the improvement of $p_{3}^{A}$ has mostly contributed to the overall improvement, i.e. $63 \%$ of the overall improvement.

$$
\text { Quantification of } C_{K}^{A \rightarrow B} \text { for } 1 \leq|K| \leq n
$$

This case is a generalisation of the previous cases, given that the improvement contribution of a set on mono-criterion performances is no more than the sum of the improvement contributions of each mono-criterion performance. The interactions being handled in the quantification of each improvement contribution, the overall improvement contribution can be written as an arithmetic sum:

$C_{K}^{A \rightarrow B}=\sum_{i \in K} C_{i}^{A \rightarrow B}$

where $C_{i}^{A \rightarrow B}$ is the improvement contribution of the monocriterion performance $p_{i}^{A}$ to the overall improvement performance $\Delta p_{O v}^{A \rightarrow B}$.

Example 3 Let us consider again the previous example; by applying (3) the contributions for improving 2 performances are the following:

$$
\begin{aligned}
& C_{23}^{A \rightarrow B}=C_{2}^{A \rightarrow B}+C_{3}^{A \rightarrow B}=0.03+0.12=0.15 \\
& C_{24}^{A \rightarrow B}=C_{2}^{A \rightarrow B}+C_{4}^{A \rightarrow B}=0.03+0.041=0.071 \\
& C_{34}^{A \rightarrow B}=C_{3}^{A \rightarrow B}+C_{4}^{A \rightarrow B}=0.12+0.041=0.161
\end{aligned}
$$

Hence, the greatest improvement contribution is obtained by improving the mono-criterion performances $p_{3}^{A}$ and $\Delta p_{O v .}^{A}=\sum_{\mathrm{i}=1}^{\mathrm{n}} w_{i}^{\prime} p_{i}^{A}$. Improving $p_{2}^{A}$ and $\Delta p_{O v}^{A}$. $=$ $\sum_{\mathrm{i}=1}^{\mathrm{n}} w_{i}^{\prime} p_{i}^{A}$ contributes less on the overall performance.
Let us note that the case where the intermediate states between A and B are not known is considered in Appendix 2. This case leads to a range of values for the improvement contribution instead of a precise value.

In the following section, let us consider how the improvement contribution quantification can be used for intelligent manufacturing control.

\section{Intelligent control using the improvement contribution}

As well as the PMS pieces of information, the improvement contribution constitutes an interactive element with a decision-maker. To be more precise, we consider here a PDCA cycle improvement and imagine that such a cycle is at the beginning (Plan step) of an iteration, namely state A (cf. assumption (A5) § 2.2). The reached performances are respectively $\vec{P}^{A}$ and $p_{O v}^{A}$. Let us consider that state B identifies the end of the iteration (Act Step) (cf. assumption (A5) $\S 2.2$ ).

From a general point of view, the improvement contribution constitutes an interacting piece of information that allows the decision-maker to fix the overall expected performance of state $\mathrm{B}$, the vector of associated mono-criterion performances, as well as to make a diagnosis about what happened between the two states, in terms of mono-criterion performance improvement. Such an improvement contribution can be a decisive piece of information in the choice of the action plans, according to the decision-maker's preferences as well as to the company context (cf. § 1). In this approach, we assume that the choice of the improvement action plan is no more than the choice of the mono-criterion performances to improve.

Let us talk more precisely about what has been developed in this paper. By assuming that the improvement cycle is at state $\mathrm{B}$, the improvement contribution can inform the decision-maker about the way this improvement has been achieved, with regards to the set of mono-criterion performances involved. The available information concerns the improvement contribution of each mono-criterion performance, separately and jointly with the others, namely which mono-criterion performance(s), has(ve) been correctly achieved and what has to be corrected. Note that these pieces of information can be used as a management tool, by rewarding or congratulating the teams involved in the improvement.

The simplified procedure described hereafter summarises the operations that cover the intelligent interaction between 
the decision-maker and the performance information provided by the considered PMS, for a given iteration of PDCA Cycle (from state A to state B).

At state A: 1 For $\vec{P}^{A} p_{O v}^{A}=C I\left(\vec{P}^{A}\right)$ according to (4). At

state B: 2. For $\vec{P}^{B}, \Delta p_{i}^{A \rightarrow B} i=1$ to $n p_{O v .}^{B}=C I\left(\vec{P}^{B}\right)$ according to (4).

and $\Delta p_{O v .}^{A \rightarrow B}=p_{O v .}^{B}-\Delta p_{O v}^{A}$.

Then: $C_{i}^{A \rightarrow B}$ is quantified according to (2).

Then: $C_{K}^{A \rightarrow B}$ is quantified according to (3).

Then: the most contributing $\Delta p_{i}^{A \rightarrow B}$ and $\Delta p_{K}^{A \rightarrow B}$ are identified.

Let us note that a similar approach can be used in the Planstep, by considering planned values rather than achieved values, as we will see in the case study described hereafter.

\section{Case study}

\section{Background}

The case study is issued from the Fournier Company (Thônes, France), a SME that produces kitchens, bathrooms and storing closets. The Fournier Company is organised into 5 manufacturing sites, which are located in the Haute-Savoie Region, in France. Such sites have been defined according to the different family products that are manufactured by the Company, namely the kitchens, the bathrooms and other storage elements. Moreover, the Company manufactures more than 850,000 items and its weekly production is 5000 pieces of furniture. The main product remains the kitchen and is made of about 10 different parts, each part containing between 10 and 50 pieces. For a kitchen, the manufacturing cycle time is between 2 and 5 weeks.

Our collaboration with the Company has been established for about fifteen years. Indeed, certified ISO 9001 since 2004, the Company initially implemented a quantified PMS according to our PMS aggregation model (see Appendix 1). Besides, the Company has also fully adopted the PDCA cycle for structuring its improvement approaches. At the beginning of 2011, the company fixed an overall performance which is related to the increase of its Business turn-over by about $20 \%$ by the end of 2013 . Throughout the 3 -year duration of the improvement project, a review was organised, each semester, thus allowing decision-maker to check each achieved improvement step, to act and to plan the following improvement. To clarify, the 4 mono-criterion performances are presented hereafter.

- 1: the Advertising level.

- 2: the Product price.

- 3: the Customer satisfaction.

- 4: the Retailers number.
The $C I$ coefficients are given in Table 1.

The top management is aware that the improvement cycle is complex and depends for a large part on the teams' involvement. Decision-maker would thus like to obtain some pieces of information that will allow him to control the improvement according to three rules:

a priori of the improvement enactment:

- (R1) plan the overall performance improvement in an as simple as possible way, by inducing as few monocriterion performance improvements as possible;

- (R2) plan the overall performance in an as regular as possible way throughout the duration of the project; $a$ posteriori of the improvement enactment:

- (R3) reward the different teams proportionally to the contribution to the overall improvement. The rewards are attributed mono-criterion performance by monocriterion performance. In the Company, each monocriterion performance improvement is under the control of one specific team.

\section{Fournier Company improvement contribution quantification}

The Company planned for December 2013 a total achievement, for the overall performance as well as for the 4 mono-criterion performances. The amount of reward is considered to be $10 \mathrm{~K}^{3}$.

The PDCA has been iterated 6 times, each iteration corresponding to a semester:

Semester 1-January-June 2011-state A to state B.

Semester 2-July-December 2011—state B to state C.

Semester 3 - January-June 2012-state C to state D.

Semester 4 - July-December 2012—state D to state E.

Semester 5 - January-June 2013-state E to state F.

Semester 6 - July-December 2013-state F to state G.

At state A: $\vec{P}^{A}=(0.6,0.2,0.55,0.35)$ and $p_{O v}^{A}$. $=$ 0.358 . In order to handle the planned values, let us introduce the following notation: $\vec{P}^{\alpha p l a n n e d}$ and $p_{O v}^{\alpha p l a n n e d}$ at state $\alpha$. Thus, according to (R2), the planned performances are respectively: $P_{O v}^{\text {Bplanned }}=0.5, P_{O v}^{C p l a n n e d}=$ $0.6, P_{O v}^{\text {Dplanned }}=0.7, P_{O v}^{\text {Eplanned }}=0.8, P_{O v}^{F \text {. }}$. $=$ 0.9, $P_{\text {Ov. }}^{\text {Gplanned }}=1$ with $\vec{P}^{\text {Gplanned }}=(1,1,1,1)$.

A priori of the improvement enactment, decision-maker looks for the break-down of the overall planned performance into the mono-criterion planned performances that satisfy (R1). This problem thus raises a priori of each iteration.

A posteriori of the improvement enactment, decisionmaker looks for information about the contribution of the

\footnotetext{
3 The exact amount cannot be communicated for confidentiality reasons.
} 
Table 2 Planned and achieved mono-criterion and overall performances during the 6 semesters

\begin{tabular}{lll}
\hline & Vector of mono-criterion performances & Overall performance \\
\hline Initial performance & $\vec{P}^{A}=(0.6,0.2,0.55,0.35)$ & $p_{\text {Ov. }}^{A}=0.358$ \\
Achieved performance & $\vec{P}^{B}=(0.6,0.51,0.55,0.35)$ & $p_{\text {Ov. }}^{B}=0.482$ \\
& $\vec{P}^{C}=(0.60,0.51,0.68,0.74)$ & $p_{\text {Ov. }}^{C}=0.594$ \\
& $\vec{P}^{D}=(0.71,0.70,0.68,0.74)$ & $p_{\text {Ov. }}^{D}=0.691$ \\
& $\vec{P}^{E}=(0.89,0.91,0.68,0.74)$ & $p_{\text {Ov. }}^{E}=0.778$ \\
& $\vec{P}^{F}=(0.89,0.91,0.93,0.87)$ & $p_{\text {Ov. }}^{F}=0.890$ \\
& $\vec{P}^{G}=(0.95,0.97,1,0.89)$ & $p_{\text {Ov. }}^{G}=0.939$ \\
& $\vec{P}^{\text {Bplanned }}=(0.6,0.56,0.5,0.35)$ & $p_{\text {Ov. }}^{\text {Bplanned }}=0.5$ \\
& $\vec{P}^{\text {Cplanned }}=(0.6,0.51,0.71,0.71) 0$ & $p_{\text {Ov. }}^{\text {Cplanned }}=0.6$ \\
& $\vec{P}^{\text {Dplanned }}=(0.72,0.72,0.68,0.74)$ & $p_{\text {Ov. }}^{\text {Dplanned }}=0.7$ \\
& $\vec{P}^{\text {Elanned }}=(0.94,0.94,0.68,0.74)$ & $p_{\text {Ov. }}^{\text {Eplanned }}=0.8$ \\
& $\vec{P}^{\text {Flanned }}=(0.89,0.91,0.92,0.92)$ & $p_{\text {Ov. }}^{\text {Fplanned }}=0.9$ \\
& $\vec{P}^{\text {Gplanned }}=(1,1,1,1)$ & $p_{\text {Ov. }}^{\text {Gplanned }}=1$ \\
\hline
\end{tabular}

mono-criterion performance improvement in order to make his diagnosis and to reward the involved team, according to (R3). This problem thus raises a posteriori of each iteration. Decision-maker has to define the reward with regards to the overall improvement cycle. The reward is thus the ratio between the mono-criterion performance improvement contribution and the overall planned improvement $\Delta p_{O v .}^{A \rightarrow \text { Gplanned }}=1-0.358=0.642$.

During our collaboration with the Fournier Company, we have handled these two decision-maker requirements throughout the 6 semesters (see Table 2). For the sake of illustration, we describe hereafter the work realised for the $1^{\text {st }}$ semester and the $2^{\text {nd }}$ semester.

\section{Improvement contribution quantification for the 1 st semester}

A priori of the iteration, let us recall that: $\vec{P}^{A}=(0.6,0.2$, $0.55,0.35)$ and $p_{O v}^{A}=0.358 . P_{O v}^{\text {Bplanned }}=0.5$. Then: $\Delta P_{O v}^{A \rightarrow \text { B }}$. $=0.142$.

In order to determine $\vec{P}^{\text {Bplanned }}, C_{K}^{A \rightarrow B}$ are quantified, beginning by $|K|=1$ according to (R1), i.e. for $p_{i}^{\text {Bplanned }}=$ 1. Then (see Fig. 3):

If $\vec{P}^{\text {Bplanned }}=(1,0.2,0.55,0.35)$ Then $C_{1}^{A \rightarrow B}=0.02$

If $\vec{P}^{\text {Bplanned }}=(0.6,1,0.55,0.35)$ Then $C_{2}^{A \rightarrow B}=0.20$

If $\vec{P}^{\text {Bplanned }}=(0.6,0.2,1,0.35)$ Then $C_{3}^{A \rightarrow B}=0.04$

If $\vec{P}^{\text {Bplanned }}=(0.6,0.2,0.55,1)$ Then $C_{4}^{A \rightarrow B}=0.07$

The only possibility to improve only one mono-criterion performance is to improve $p_{2}^{A}$. Indeed, the minimum value to have $P_{O v}^{B \text { planned }}=0.5$, i.e. $\Delta P_{O v}^{A \rightarrow \text { Bplanned }}=0.142$, is to have $C_{i}^{A \rightarrow B} \geq 0.142$, i.e. $\Delta p_{2}^{A \rightarrow B}=0.36$ leading to $p_{2}^{\text {Bplanned }}=0.56$.

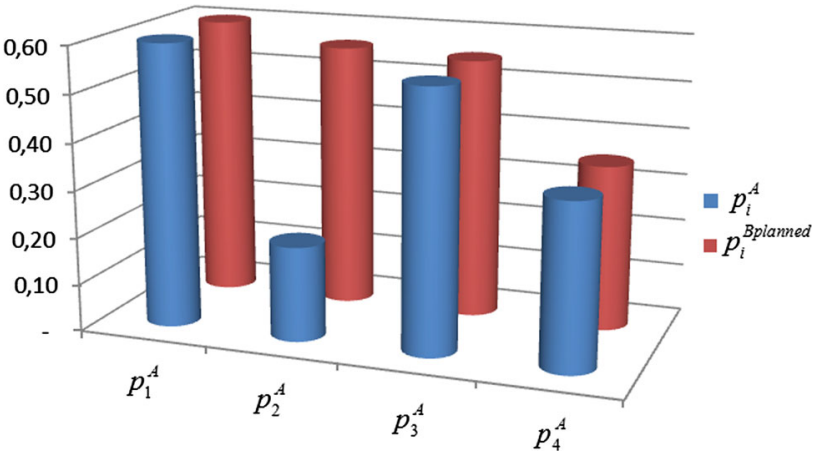

Fig. $3 \vec{P}^{A}$ and $\vec{P}^{\text {Bplanned }}$ for the $1^{\text {st }}$ semester

A posteriori of the iteration, the achieved results are $\vec{P}^{B}=$ $(0.6,0.51,0.55,0.35)$ and $p_{O v}^{B}=0.482$ (with $\Delta P_{O v}^{A \rightarrow B}=$ 0.124 ) that is a little lower than the plan value.

According to (R3):

$C_{1}^{A \rightarrow B}=0$ then reward $=\frac{0}{0.0642} \times 10000 €=0 €$

$C_{2}^{A \rightarrow B}=0.124$ then reward $=\frac{0.124}{0.0642} \times 10000 €=1903 €$

$C_{3}^{A \rightarrow B}=0$ then reward $=\frac{0}{0.0642} \times 10000 €=0 €$

$C_{4}^{A \rightarrow B}=0$ then reward $=\frac{0}{0.0642} \times 10000 €=0 €$

Moreover, the $C_{2}^{A \rightarrow B}$ is the only contributing monocriterion performance while $C_{1}^{A \rightarrow B}, C_{2}^{A \rightarrow B}$ and $C_{4}^{A \rightarrow B}$ do not contribute to the overall improvement (see Fig. 4).

\section{Improvement contribution quantification for the 2nd semester}

A priori of the iteration, let us recall that: $\vec{P}^{B}=(0.6,0.51$, $0.55,0.35)$ and $p_{O v}^{B}=0.482 p_{O v}^{\text {Cplanned }}=0.6$. Then: $\Delta p_{\text {Ov. }}^{B \rightarrow \text { Cplanned }}=0.118$. 


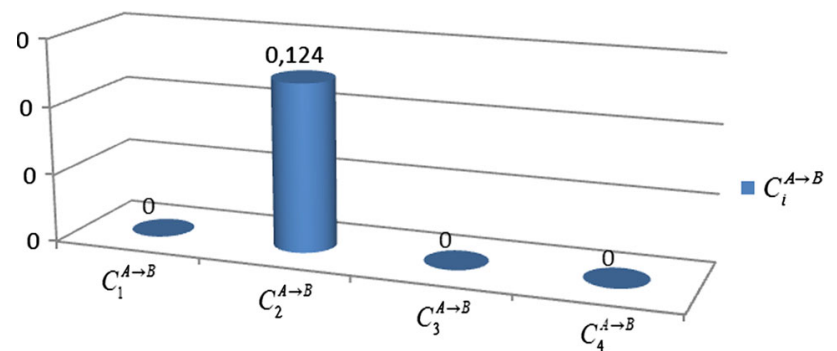

Fig. 4 Values of $C_{i}^{A \rightarrow B}$ for the $1^{\text {st }}$ semester

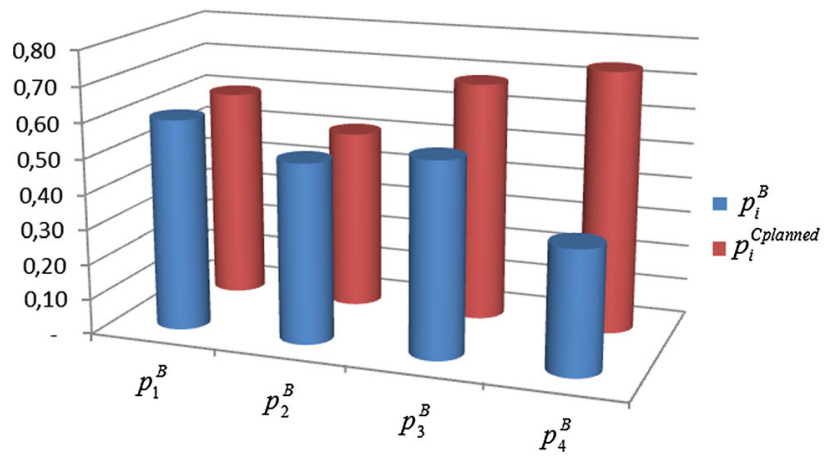

Fig. $5 \vec{P}^{B}$ and $\vec{P}^{\text {Cplanned }}$ for the $2^{\text {nd }}$ semester

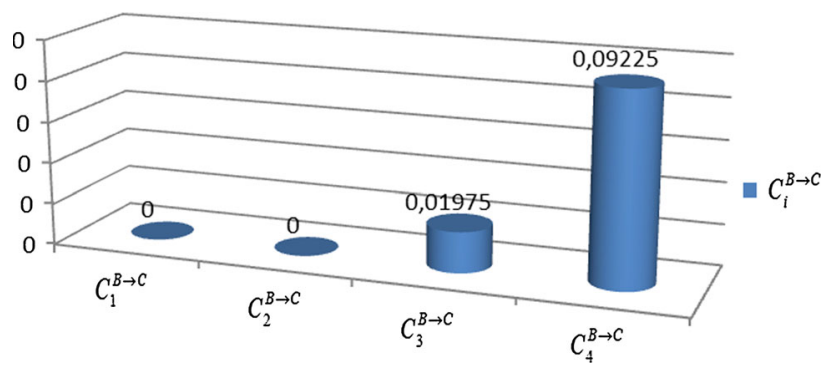

Fig. 6 Values of $C_{i}^{B \rightarrow C}$ for the 2 nd semester

In order to determine $\vec{P}^{\text {Cplanned }}, C_{K}^{B \rightarrow C}$ are quantified, beginning by $|K|=2$ according to (R1), i.e. for $p_{i \in K}^{\text {Cplanned }}=$ 1. Then (see Fig. 5):

If $\vec{P}^{\text {Cplanned }}=(1,1,0.55,0.35)$ Then $C_{12}^{B \rightarrow C}=0.216$

If $\vec{P}^{\text {Cplanned }}=(1,0.51,1,0.35)$ Then $C_{13}^{B \rightarrow C}=0.144$

If $\vec{P}^{\text {Cplanned }}=(1,0.51,0.55,1)$ Then $C_{14}^{B \rightarrow C}=0.086$

If $\vec{P}^{C \text { planned }}=(0.6,1,1,0.35)$ Then $C_{23}^{B \rightarrow C}=0.12$

If $\vec{P}^{\text {Cplanned }}=(0.6,1,0.55,1)$ Then $C_{24}^{B \rightarrow C}=0.142$

If $\vec{P}^{\text {Cplanned }}=(0.6,0.51,1,1)$ Then $C_{34}^{B \rightarrow C}=0.223$

There are many possibilities to improve only 2 monocriterion performances. Decision-maker has chosen to improve $p_{3}^{B}$ and $p_{4}^{B}$ because they are the most contributing mono-criterion performances. For instance, $\vec{P}^{\text {Cplanned }}=$ $(0.6,0.51,0.71,0.71)$ where $p_{3}^{\text {Cplanned }}$ and $p_{4}^{\text {Cplanned }}$ are balanced.
A posteriori of the iteration, $\vec{P}^{C}=(0.60,0.51,0.68$, $0.74)$ and $p_{O v .}^{C}=0.594$. Then: $\Delta P_{O v}^{B \rightarrow C}=0.114$. Decisionmaker knows that $p_{3}^{B}$ has been first improved and then $p_{4}^{B}$.

According to (R3):

$$
\begin{aligned}
& C_{1}^{B \rightarrow C}=0 \text { then reward }=\frac{0}{0.0642} \times 10000 €=0 € \\
& C_{2}^{B \rightarrow C}=0 \text { then reward }=\frac{0}{0.0642} \times 10000 €=0 € \\
& C_{3}^{B \rightarrow C}=0.01975 \text { then reward }=\frac{0.01975}{0.642} \times 10000 €=
\end{aligned}
$$
$552 €$

$C_{4}^{B \rightarrow C}=0.0533$ then reward $=\frac{0.09225}{0.0642} \times 10000 €=$ $2577 €$

$C_{4}^{B \rightarrow C}$ is the most contributing mono-criterion performance (around $80 \%$ ) while $C_{3}^{A \rightarrow B}$ contributed only at $20 \%$ to the overall improvement (see Fig. 6).

\section{Conclusion}

In a manufacturing overall performance improvement context, where the overall performance is multi-criteria, we have defined the improvement contribution concept to be information about the impact that mono-criterion performance improvements have on the improvement of the overall performance.

By assuming that interactions exist between the involved mono-criterion performances, the Choquet Integral (CI) aggregation performance model that has been previously developed has been the basic of this work. Thus, a quantification of the improvement contribution, for one or several mono-criterion performance improvement(s) has been proposed. The developed idea was to consider the CI performance aggregation model for highlighting the interaction and to use it in the same logic as the WAM operator, for quantifying the mono-criterion performance improvement contribution.

The relevance of the improvement contribution concept has been shown through an intelligent procedure that makes it possible to create interaction between decision-maker and the performance improvement pieces of information, in particular the improvement contribution information. With regards to industrial practice, the usefulness of the improvement contribution concept has been already confirmed through its deployment in a case submitted by the Fournier Company concerning its Business turn-over performance improvement.

The main perspectives concern on the one hand the integration of the additional functionalities in the management process, i.e. what and when the different improvement contributions must be computed and how they can be used for structuring the improvement and motivating the involved teams. In this sense, works are in-progress concerning the formal framework of quantification of the improvement contribution. 


\section{Appendix 1: Fundamentals of the choquet integral (CI)}

\section{The initial form of the CI operator}

To deal with the involved performance interactions, the $\mathrm{CI}$ operators provide powerful aggregation operators. To be more precise, the CI operators can be seen as an extension of the WAM operators, and are able to consider not only weights for mono-criterion performances but in addition weights for any subset of performances (Grabisch 1996; Labreuche and Grabisch 2003). However, such a model requires $\left(2^{n}-2\right)$ parameters in order to take into account the weight of each possible subset of performances (from 1 to $n$ corresponding mono-criterion performances). This represents a huge identification task in practice. Hence, more tractable models have been introduced with a sufficient information level, e.g., the 2-additive CI that only considers weights for pairs of performances (called in that case interactions between 2 (Grabisch 1997). These operators are a kind of compromise between complexity and richness of the model, since only $\left(\frac{n(n+1)}{2}\right)$ parameters are required. We propose hereafter notation in accordance with the PMS ones, where $p_{i}^{A}$ is a given monocriterion performance (defined on $[0,1]$ ) at the state A and $p_{j}^{A}$ is another one mono-criterion performance at the same state A.

2 kinds of coefficients are involved in a 2-additive CIbased aggregation model:

- the weight of each mono-criterion performance in relation to the overall performance by the so-called Shapley coefficients $\phi_{\mathrm{i}}^{\prime} s$, that satisfy the condition $\sum_{\mathrm{i}=1}^{\mathrm{n}} \phi_{\mathrm{i}}=1$ (which is a quasi-natural condition);

- the interaction coefficient of any pair of mono-criterion performances $p_{i}^{A}$ and $p_{j}^{A}$, denoted $I_{\mathrm{ij}} . I_{\mathrm{ij}}$ ranges within the interval $[-1 ; 1]$. The " -1 "value expresses a complete redundancy between 2 mono-criterion performances, whereas the " -1 " value means complete complementarity of these mono-criterion performances.

Thus, the overall performance $p_{O v}^{A}$. aggregated by a 2additive $\mathrm{CI}$ is given by (Grabisch 1997):

$$
\begin{aligned}
& p_{O v .}^{A}=C I\left(\vec{P}^{A}\right)=\sum_{\mathrm{i}=1}^{\mathrm{n}}\left(\phi_{i}-\frac{1}{2} \sum_{j \neq i}\left|I_{i j}\right|\right) p_{i}^{A} \\
& +\sum_{I_{i j}>0}\left|I_{i j}\right| \min \left(p_{i}^{A}, p_{j}^{A}\right)+\sum_{I_{i j}<0}\left|I_{i j}\right| \max \left(p_{i}^{A}, p_{j}^{A}\right)
\end{aligned}
$$

with the property $\left(\phi_{\mathrm{i}}-\frac{1}{2} \sum_{j \neq i}\left|I_{\mathrm{ij}}\right|\right) \geq 0, \forall i \in[1, n]$ in order to ensure the monotonicity of the aggregation operator.

Note that the mechanism of determination of the Shapley and interaction coefficients is based on the expertise of the decision-maker. The handling of this expertise may be carried out with the MACBETH method in a coherent way according to the measurement theory (Krantz et al. 1971). Readers interested in the details of this method can find more information in (Clivillé et al. 2007, Bana e Costa et al. 2012).

Example 4 As an illustration of the CI performance aggregation model, let us consider the case study of $\S 5$ which involves 4 mono-criterion performances. Thus, by applying (4) to this particular case, the overall performance associated to state $p_{O v}^{A}$. becomes:

$$
\begin{aligned}
& p_{O v .}^{A}=0.05 p_{1}^{A}+0.1 p_{2}^{A}+0.075 p_{3}^{A}+0.025 p_{4}^{A} \\
& \quad+0.3 \min \left(p_{1}^{A}, p_{2}^{A}\right)+0.2 \min \left(p_{1}^{A}, p_{3}^{A}\right) \\
& \quad+0.25 \min \left(p_{3}^{A}, p_{4}^{A}\right)
\end{aligned}
$$

\section{The piecewise linear form of the CI operator}

By examining (4), one can see that it is possible to eliminate the $\min$ and $\max$ operators by considering the ranking of any pair of performances $\left(p_{i}^{A}, p_{j}^{A}\right)$ :

- When $p_{i}^{A}>p_{j}^{A}$, the term $\sum_{I_{i j}>0}\left|I_{i j}\right| \min \left(p_{i}^{A}, p_{j}^{A}\right)+$ $\sum_{I_{i j}<0}\left|I_{i j}\right| \max \left(p_{i}^{A}, p_{j}^{A}\right)$ of (4) becomes $\sum_{I_{i j}>0}\left|I_{i j}\right| p_{j}^{A}+$ $\sum_{I_{i j}<0}\left|I_{i j}\right| p_{i}^{A}$; and we have in this case $\Delta p_{O v}^{A}$. $=$ $\sum_{\mathrm{i}=1}^{\mathrm{n}} w_{i}^{\prime} p_{i}^{A}$

with

$$
\begin{aligned}
w_{i}^{\prime} & =\phi_{i}-\frac{1}{2} \sum_{j \neq i}\left|I_{i j}\right|+\sum_{I_{i j}<0}\left|I_{i j}\right| \\
& =\phi_{i}-\frac{1}{2} \sum_{I_{i j}>0}\left|I_{i j}\right|+\frac{1}{2} \sum_{I_{i j}<0}\left|I_{i j}\right|
\end{aligned}
$$

- When $p_{i}^{A}<p_{j}^{A}$, the term $\sum_{I_{i j}>0}\left|I_{i j}\right| \min \left(p_{i}^{A}, p_{j}^{A}\right)+$ $\sum_{I_{i j}<0}\left|I_{i j}\right| \max \left(p_{i}^{A}, p_{j}^{A}\right)$ of (4) becomes $\sum_{I_{i j}>0}\left|I_{i j}\right| p_{i}^{A}+$ $\sum_{I_{i j}<0}\left|I_{i j}\right| p_{j}^{A}$; and we have $\Delta p_{O v}^{A}=\sum_{\mathrm{i}=1}^{\mathrm{n}} w_{i}^{\prime \prime} p_{i}^{A} \quad \Delta p_{O v}^{A}$. $=\sum_{\mathrm{i}=1}^{\mathrm{n}} w_{i}^{\prime \prime} p_{i}^{A}$ with

$$
\begin{aligned}
w_{i}^{\prime \prime} & =\phi_{i}-\frac{1}{2} \sum_{j \neq i}\left|I_{i j}\right|+\sum_{I_{i j}<0}\left|I_{i j}\right| \\
& =\phi_{i}-\frac{1}{2} \sum_{I_{i j}>0}\left|I_{i j}\right|+\frac{1}{2} \sum_{I_{i j}>0}\left|I_{i j}\right|
\end{aligned}
$$

Example 5 Let us consider again the example 4 corresponding also to example 3 in $\S 3.3 .2$ 
When $p_{1}^{A}>p_{2}^{A}>p_{4}^{A}>p_{3}^{A}$ then $w_{1}=0.05, w_{2}=$ $0.3+0.1=0.4, w_{3}=0.075+0.2+0.25=0.525, w_{4}=$ 0.025 .

When $p_{1}^{\alpha}>p_{2}^{\alpha}>p_{3}^{\alpha}>p_{4}^{\alpha}$ then

$w_{1}=0.05, w_{2}=0.3+0.1=0.4$,

$w_{3}=0.075+0.2=0.275, w_{4}=0.025+0.25=0.275$

When $p_{2}^{A}>p_{1}^{A}>p_{3}^{A}>p_{4}^{A}$ then

$w_{1}=0.05+0.3=0.35, w_{2}=0.1$,

$w_{3}=0.075+0.2=0.275, w_{4}=0.025+0.25=0.275$

This result can be generalized to any Choquet integral beyond the 2-additive ones (Montmain et al. 2005).

\section{Appendix 2: Improvement contribution quantification without knowledge of the intermediate states}

In $\S 3.3$, the improvement contribution quantification is based on the knowledge of the performance ranking changes. When it is not the case, i.e. the intermediate states between states A and B are not known, the preceding approach is not applicable. Nevertheless, it is possible to quantify the lower and upper values of the improvement contribution, by maximising, and respectively minimising, versus $p_{i}^{A}$, the non-linear part of the CI, i.e. $\sum_{I_{i j}>0}\left|I_{i j}\right| \min \left(p_{i}^{A}, p_{j}^{A}\right)+$ $\sum_{I_{i j}<0}\left|I_{i j}\right| \max \left(p_{i}^{A}, p_{j}^{A}\right) \quad$ leading thus to $C_{i}^{A \rightarrow B u p}$ and $C_{i}^{A \rightarrow B \text { low }}$.

Example 6 Let us consider again the preceding example where the mono-criterion performances improve from $\vec{P}^{A}=$ $(0.6,0.55,0.2,0.35)$ to $\vec{P}^{B}=(0.6,0.7,0.5,0.5)$. Therefore the upper and lower improvement contributions are quantified as follows:

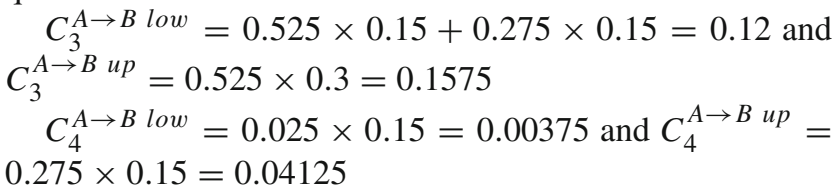

In this case, whatever the improvement way the contribution of improving $p_{3}^{A}$ notation is much higher than improving $p_{4}^{A}$ notation.

\section{References}

Bana e Costa, C., \& De Corte, J. M. (2012). MACBETH. International Journal of Information Technology and Decision Making, 11(02), 359-387.
Berrah, L., Montmain, J., Mauris, G., \& Clivillé, V. (2011). Optimising industrial performance improvement within a quantitative multicriteria aggregation framework. International Journal of Data Analysis Techniques and Strategies, 3(1), 42-65.

Bititci, U. S. (2001). Strategy management through quantitative modelling of performance measurement systems. International Journal of Production Economics, 69, 137-147.

Bosch-Mauchand, M., Siadat, A., Perry, N., \& Bernard, A. (2012). VCS: value chains simulator, a tool for value analysis of manufacturing enterprise processes (a value-based decision support tool ). Journal of Intelligent Manufacturing, 23(4), 1389-1402.

Bourne, M., Mills, J. F., Wilcox, M., Neely, A. D., \& Platts, K. W. (2000). Designing, implementing and updating performance measurement systems. International Journal of Operations Production management, 20(7), 754-771.

Clivillé, V., Berrah, L., \& Mauris, G. (2007). Quantitative expression and aggregation of performance measurements based on the MACBETH multi-criteria method. International Journal of Production Economics, 105(1), 171-189.

Gallasso, F., Ducq, Y., Lauras, M., Gourc, D., \& Camara, M. (2016). A method to select a successful interoperability solution through a simulation approach. Journal of Intelligent Manufacturing, 27(1), 217-229.

Ghalayini, A. M. (1996). The changing basis of performance measurement. International Journal of Operations and Production Management, 16(8), 63-80.

Ghalayini, A. M., Noble, J. S., \& Crowe, T. J. (1997). An integrated dynamic performance measurement system for improving manufacturing competitiveness. International Journal of Production Economics, 48(3), 207-225.

Globerson, S. (1985). Issues in developing a performance criteria system for an organisation. International Journal of Production Research, 23(4), 639-646.

Grabisch, M. (1996). The application of fuzzy integrals in multicriteria decision making. European Journal of Operational Research, 89, 445-456.

Grabisch, M. (1997). k-ordered discrete fuzzy measures and their representation. Fuzzy Sets and Systems, 92, 167-189.

Imai, M. (1986). Kaizen: The key to Japan's competitiveness. New York: Mac Graw-Hill Higher Education.

Johnson, H. T. (1975). Management accounting in early integrated industry - E. I. Dupont de Nemours Powder Company 1903-1912. Business History Review, Summer, 48(2), 184-204.

Kaplan, R. S., \& Norton, D. P. (1992). The balanced scorecard: Measures that drive performance. Harvard Business Review, 70(1), 71-79.

Kaplan, R. S., \& Norton, D. P. (1996). The Balanced Scorecard: Translating Strategy into Action. Boston: Harvard Business School Press.

Kocaoğlu, B., Gülsün, B., \& Tanyaş, M. (2013). A SCOR based approach for measuring a benchmarkable supply chain performance. Journal of Intelligent Manufacturing, 24(1), 113-132.

Krantz, D. H., Luce, R. D., Suppes, P., \& Tversky, A. (1971). Foundations of measurement (Vol. 1)., Additive and Polynomial Representations Cambridge: Academic Press.

Labreuche, C., \& Grabisch, M. (2003). The Choquet integral for the aggregation of interval scales in multicriteria decision making. Fuzzy Sets and Systems, 137, 11-26.

Montmain, J., Mauris, G., \& Akharraz, A. (2005). Elucidation and decisional risk in a multicriteria decision based on a Choquet integral aggregation- a cybernetic framework. International Journal of Multi-Criteria Decision Analysis, 13(5-6), 239-258.

Neely, A., Gregory, M., \& Platts, K. (1995). Performance measurement system design: a literature review and research agenda. International Journal of Operations and Production Management, 48(4), 80-116. 
Nudurupati, S. S., Bititci, U. S., Kumar, V., \& Chan, F. T. S. (2011). State of the art literature review on performance measurement. Computers and Industrial Engineering, 60(2), 279-290.

Ohno, T. (1988). Toyota production system: Beyond large-scale production. Boca Raton: Productivity Press.

Ounnar, F., \& Pujo, P. (2012). Pull control for job shop: Holonic manufacturing system approach using multicriteria decision-making. Journal of Intelligent Manufacturing, 23, 141-153.

Oxford dictionary, (2016). http://www.oxforddictionaries.com/defini tion/english/contribution.

Oztemel, E. (2010). Intelligent manufacturing systems. Artificial Intelligence techniques for networked manufacturing enterprises management (pp. 1-41). Berlin: Springer.

Saaty, T. (2000). Fundamentals of the analytic hierarchy process. Pittsburgh: RWS Publications.

Schneidermann, A. M. (1988). Setting quality goals. Quality Progress, $21,51-75$.
Shah, L. A., Etienne, A., Siadat, A., \& Vernadat, F. (2016). Decisionmaking in the manufacturing environment using a value-risk graph. Journal of Intelligent Manufacturing, 27(3), 617-630.

Singh, S., Olugu, E., Musa, S., \& Mahat, A. (2015). Fuzzy-based sustainability evaluation method for manufacturing SMEs using balanced scorecard framework. Journal of Intelligent Manufacturing. doi:10.1007/s10845-015-1081-1.

Suwignjo, P., \& Bititci, U. S. (2000). Quantitative models for performance measurement system. International Journal of Production Economics, 64, 231-241.

Waggoner, D. B., Neely, A. D., \& Kennerley, M. P. (1999). The forces that shape organizational performance measurement systems: An interdisciplinary review. International Journal of Production Economics, 60-61, 53-60. 